\title{
Damage Threshold of Borosilicate Glass Under Plate Impact
}

\author{
Sidney Chocron ${ }^{1}$ Darrel D. Barnette ${ }^{1} \cdot$ Timothy J. Holmquist $^{1}$ • \\ Charles E. Anderson Jr. ${ }^{1} \cdot$ Rory P. Bigger $^{1} \cdot$ Thomas Z. Moore $^{1}$
}

Received: 14 October 2015/Accepted: 25 January 2016/Published online: 1 February 2016

(C) Society for Experimental Mechanics, Inc 2016

\begin{abstract}
Symmetric plate-impact tests of borosilicate glass projectiles into borosilicate glass targets were performed at stress levels from 0.7 to $2 \mathrm{GPa}$ (impact velocities from 116 to $351 \mathrm{~m} / \mathrm{s}$ ). As far as the authors know this range of velocities has not been explored in the literature previously. The tests used an ultra-high-speed camera to record shock and failure propagation. The velocity of the back of the target was also recorded with a photon Doppler velocimeter (PDV). The images clearly show the shock wave and its propagation at the speed expected. The reflected tensile wave is also apparent. What the authors interpret as the failure wave/front is clearly observed and appears at stress levels of $0.8 \mathrm{GPa}$ (velocities as low as $130 \mathrm{~m} / \mathrm{s}$ ). The images apparently show failure nucleation sites that trail the shock wave. These seem to be closer to the shock wave for higher speeds. A possibility is that the defects open faster at higher impact velocity while at lower velocities they are still present but remain undetected. Interestingly, even though the failure wave is clearly seen, the PDV never detected the expected recompression wave. The reason might be that at these low impact velocities the recompression wave is too small to be seen and is lost in the noise. This, in the past, may have confused researchers by thinking that the failure wave was not present at these low impact velocities. This work also presents a new way to interpret the signals from the PDV. By letting part of the signal travel through the target and reflect on the impact side, it is possible to see the PDV signal decrease in intensity with time. This would be consistent with having damage in the
\end{abstract}

Sidney Chocron

schocron@swri.edu

1 Southwest Research Institute, 6200 Culebra Rd, San Antonio, TX 78238, USA interior of the specimen, something not straightforward to confirm through just high-speed photography.

Keywords Plate impact - Borosilicate glass - Ultra high speed imaging $\cdot$ Photon doppler velocimetry

\section{Introduction}

Shock waves in glass have been the subject of intense research since the 1950s [1]. Shock waves are usually introduced in the glass specimens via explosive or plateimpact techniques. Three themes have sparked a lot of interest in relation to the plate-impact experiment: (1) shock compression and the Hugoniot elastic limit (HEL) of glass, (2) tensile waves and spall strength, and (3) failure "waves" or failure "fronts" in glass.

Cagnoux [2], starting in 1982, performed tests on borosilicate glass similar in nature and geometry to the tests presented in this paper. Cagnoux tests confirmed, see [3], previous work that Kanel et al. [4] performed on a borosilicate glass called K-8. Cagnoux had a very interesting observation: the apparent spall strength in glass decreased with lower impact velocities and thicknesses. In particular, he measured spall stresses of 1.05, 1.4, and $2.2 \mathrm{GPa}$ for experiments that reached compression stresses of 3.2, 3.8, and 5.2 GPa, respectively. He also reported an elastic response at $0.7 \mathrm{GPa}$, which we will show, was confirmed by our experiments, reported herein. In a followon paper, Cagnoux [5] showed that the HEL of borosilicate was $7.5 \mathrm{GPa}$ which included a non-linear range from 0 to $3 \mathrm{GPa}$, probably due to densification, followed by an approximately linear behavior from 3 to $7.5 \mathrm{GPa}$. This is in nominal agreement with the HEL reported by Alexander et al. [6] of $8.7 \mathrm{GPa}$, although Alexander adjusted the HEL 
value to $5.3 \mathrm{GPa}$ due to the softening of the bulk and shear moduli.

Yeshurun et al. [7] tested Pyrex between 300 and $1300 \mathrm{~m} / \mathrm{s}$ and observed no spall on impacts below the HEL and almost negligible spall strength above the HEL, which was found to be $7.8 \mathrm{GPa}$, similar to the value found by Cagnoux. The name Pyrex is, in this context, synonym of borosilicate glass manufactured by Corning.

Kanel et al. [8] explained an earlier-than-expected wave reflection (recompression) by introducing the concept of failure "wave". This wave is a failure front, moving at 2-5 km/s [9], that trailed the compressive shock wave and created free surfaces inside the material reflecting the tensile wave coming from the rear surface of the target. Across the failure front the properties of the glass would vary dramatically [10], e.g., loss of tensile strength, lower impedance and sound speed, opacity, etc. This failure wave could explain the apparent contradictory observation of the spall strength by Cagnoux and Yeshurun: if the spall plane is located behind the failure front, where the material is weaker, then a smaller or negligible strength would be observed, as seen by Cagnoux. On the other hand, if the spall plane is on the intact side then a very large spall strength should result.

Brar et al. [11], using a systematic approach to fail sodalime glass in front and behind the failure front, were unable to spall Pyrex glass below 5.2 GPa, inferring that a failure wave was not produced below that value.

As far as the authors know, the first and only effort to perform ultra-high speed imaging of the compressive failure front in plate-impact of soda-lime and borosilicate glass was by Bourne et al. [12, 13]. The shock waves are clearly observed in the pictures as well as a very dark failure front moving at velocities that depend on the impact speed. The approximate velocities for Pyrex were $3.4-3.8 \mathrm{~km} / \mathrm{s}$. For soda-lime glass the failure appeared to nucleate at a few sites and then coalesce while for borosilicate the image is cleaner and the failure front sharper and faster. Interestingly, the failure front was still present at velocities as low as $250 \mathrm{~m} / \mathrm{s}$, velocities that induced a stress level of one-third the HEL, i.e. in the elastic range. Nucleation sites were much smaller in borosilicate than in soda lime. Bourne et al. [10] later confirmed the presence of the failure waves by introducing lateral gages and looking at the two-wave structure of the signal [10].

Espinosa et al. [14] measured a spall strength of only $0.4 \mathrm{GPa}$ behind the failure front for soda-lime glass shocked to $7.5 \mathrm{GPa}$. The authors also claimed, based on experiment 7-0892, that the spall strength in front of the failure wave was $3.0 \mathrm{GPa}$. The same test was apparently reported in [15], which referenced Rosenberg et al. [16]. This last reference does mention test 8-0892 (presumably a typo on the first number) and only reports the $3.0 \mathrm{GPa}$ as a lower limit to the spall strength. In fact, tests performed by Rosenberg et al. [16] in the same paper but with foam behind the glass instead of PMMA produced tensile waves of up to $5.0 \mathrm{GPa}$ without spalling the glass. In his work, Espinosa also confirmed that the failure wave initiates at the impact surface propagating to the interior of the sample, in agreement with Kanel but in disagreement with the "nucleation, growth, and coalescence" behind the shock wave observed by Bourne [12], [13]. Ginzburg and Rosenberg [17] showed that the sound speed and impedance behind and in front of the failure front in glass are the same. This is possible if the glass is still compact behind the failure front. A similar observation on borosilicate is that of Chocron et al. [18], where borosilicate was heavily damaged by applying load cycles under confinement and the elastic constants for both damaged and undamaged material, were shown to be close.

Tolman et al. [19], using a bar impact technique and a PDV on the free end determined a spall strength for borosilicate between 0.6 and $1.1 \mathrm{GPa}$, depending on the impact velocity. Tolman explains in his paper that measured in this way the spall strength is an extrinsic property because of the influence of the surface-initiated flaws.

Partom [20] postulated the existence of a failure wave based on the experimental results of Kanel et al. Partom was able to reproduce experimental wave profiles of 1-D planar impact of soda-lime [21] and borosilicate glass [10], including the recompression signal. However, other researchers have interpreted the failure phenomenon to be one of crack coalescence and growth (e.g., Brar and Clifton, as mentioned in Anderson et al. [22]) and refer to a "failure front" and/or "failure kinetics" instead of a "failure wave."

Numerical simulations of a laser-shock experiment by Holmquist and Wereszczak permitted an estimate of the spall strength of intact borosilicate glass [23] for very short pulses. The experiment consisted of a laser shock, modeled as a nominally triangular pulse $21 \mathrm{~ns}$ in duration with a peak stress of $5.1 \mathrm{GPa}$. This laser shock resulted in a spall interior to the glass specimen with no rear or surface damage (higher pressures resulted in surface damage, while there was no damage for lower peak pressures). A spall strength of approximately 1.2-1.3 GPa produced failure in the simulations at approximately the correct depth with no rear or surface damage.

In summary the main conclusions from the literature survey are:

- So far, the spall strength of intact borosilicate glass has not been successfully measured by the pullback technique on a plate-impact test. Only a minimum spall strength has been inferred from tests that were not successful in spalling the glass. Brar et al. [11] were not able to spall the glass with stresses of up to $5 \mathrm{GPa}$; Kanel [24] tested the glass at $6.8 \mathrm{GPa}$ and did not see a spall signal either. 
- Failure fronts are observed at shock stresses well below the HEL [25].

High-speed photography, spall, shock, and failure propagation in glass were selected as the challenge for this research effort. As described in the literature review above, many questions remained open, for example, on the nature of the failure front: when it is first produced, why it is produced at impact velocities below the HEL, what is the propagation speed, and if it is a phenomenon originated on the surface or in the interior of the specimen.

A systematic study impacting glass at low velocities from 116 to $351 \mathrm{~m} / \mathrm{s}(0.7-2 \mathrm{GPa})$ has been performed. The tests have been recorded with an ultra-high-speed camera to observe the shock and failure propagation. The velocity of the back of the target has also been recorded with a photon Doppler velocimeter (PDV). This velocity is intimately related to the stresses and shock wave that travel in the specimen.

\section{Materials and Methods}

\section{Glass Material}

The borosilicate glass was acquired from Swift glass as Borofloat $33^{\circledR}$. The glass composition published by the manufacturer is: $81 \% \mathrm{SiO}_{2}, 13 \% \mathrm{~B}_{2} \mathrm{O}_{3}, 4 \% \mathrm{Na}_{2} \mathrm{O} / \mathrm{K}_{2} \mathrm{O}$, $2 \% \mathrm{Al}_{2} \mathrm{O}_{3}$. The geometry of the specimen is shown in Fig. 1. Swift glass machined the projectile and targets; both were made of the same material. Front and back of the projectile and target were polished and transparent. The flat planes machined and polished on the side of the target are the "windows" being used by the ultra-high-speed camera to record images of the interior of the specimen. The projectile diameter was designed slightly larger than that of the target. This extra space was used on the target side to place shorting pins aligned with the target that allowed measuring planarity and triggering the different data acquisition equipment.

The glass density is $2.2 \mathrm{~g} / \mathrm{cm}^{3}$ and the sound speed $5.56 \mathrm{~km} / \mathrm{s}$. The projectile thickness, as measured in the specimen was $3.94 \mathrm{~mm}$ and the target thickness was $8.12 \mathrm{~mm}$. Properties of Borofloat 33 are given by Wereszczak and Anderson [26].

\section{Experimental Set-Up}

All the tests were performed with SwRI's 50-mm gas gun that ends in a chamber containing the target package. The chamber is evacuated before performing the test. Figure $2 \mathrm{a}$ shows the technique to ensure alignment of target and projectile. The sabot is placed in the barrel with the projectile glued on its end. The projectile is butt up against the target so at this stage we have perfect planarity. The sabot and projectile are then pulled in the barrel and the shorting pins placed around the target. In Fig. $2 \mathrm{~b}$ the target holder is seen from the back showing the (black) cables from the shorting pins and the yellow cables coming from the PDV probes. The two PDV probes were used to measure the impact velocity of the projectile and the particle velocity of the back surface of the glass target.

The following diagnostics were used during the testing:

(1) PDV An NSTec PDV system, see Ref. [27], was used to measure velocity on the back surface of the target specimens. PDV is a non-contact, stand-off measurement technique that uses laser interferometry to acquire velocities for a single point on a surface at magnitudes up to $9 \mathrm{~km} / \mathrm{s}$ and at measurement rates in excess of $500 \mathrm{MHz}$. Laser light at $1550 \mathrm{~nm}$ is directed at the measurement through a collimated fiber. Reflected light is converted to voltage and the resulting RF signal is acquired on a

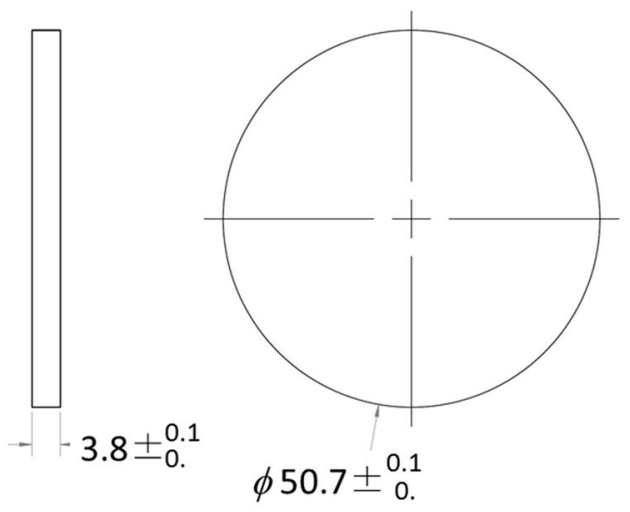

(a)

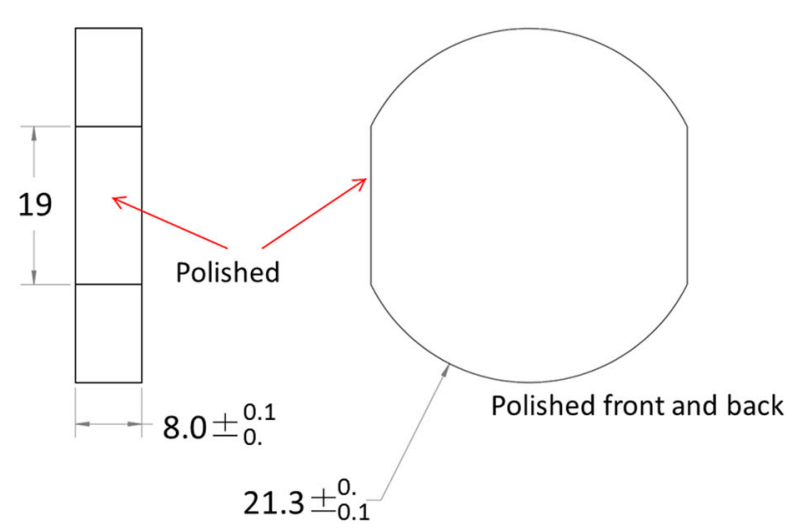

(b)

Fig. 1 Drawings of the a impactor, $\mathbf{b}$ target. All dimensions are in millimeters 

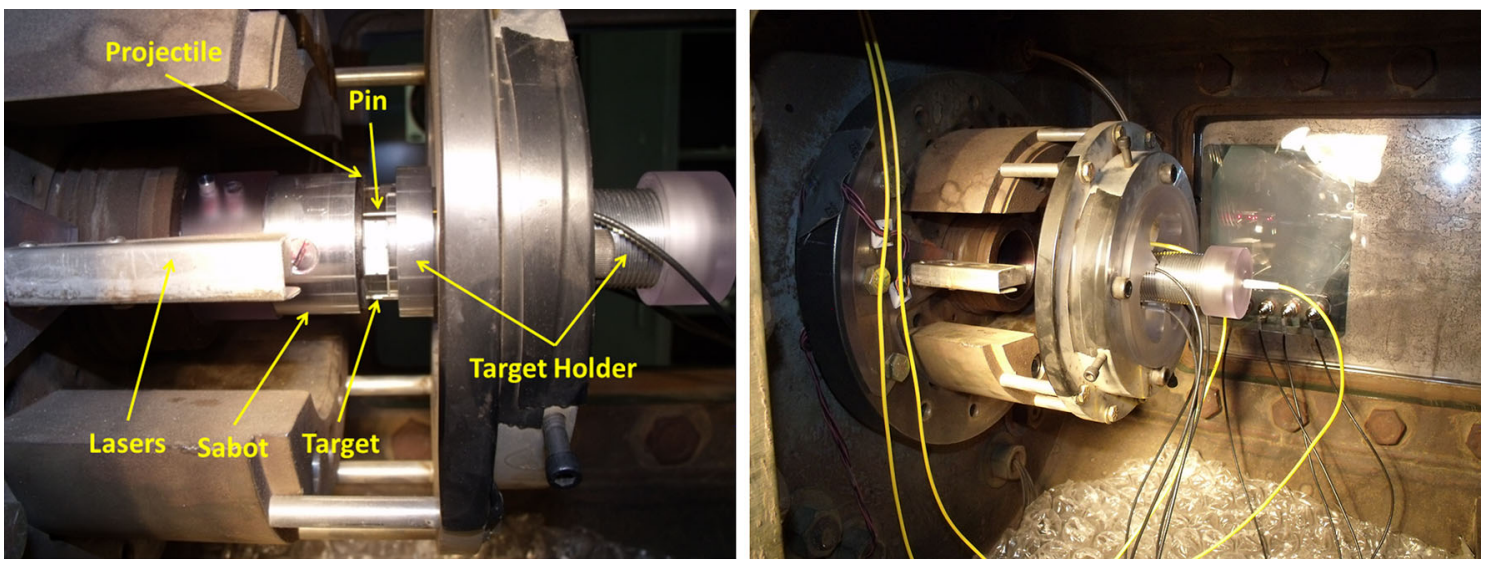

Fig. 2 a View of the sabot, projectile, target, and pins during alignment. b View from the back showing the holder and location of pins and PDV probes

$4 \mathrm{GHz}$ bandwidth, 4 channel Tektronix DPO70404 oscilloscope capable of recording at $25 \mathrm{GHz}$ (GS/s). The digitized RF signal is converted to velocity via discrete Fourier transform (i.e. a spectrogram) in post-processing software. The typical laser power setting was $1.0 \mathrm{~W}$, providing signal voltages of approximately $\pm 500 \mathrm{mV}$. Data were acquired at 6.25 or $12.5 \mathrm{GHz}$ (providing a maximum resolvable velocity of 2.4 and $4.8 \mathrm{~km} / \mathrm{s}$ respectively) over a $10 \mu$ s duration, triggered by activation of a crush-pin slightly above the surface of the target. The data acquisition settings were sufficient to discretize changes in velocity of $1.2 \mathrm{~m} / \mathrm{s}$ at $1.3 \mathrm{~ns}$ intervals.

(2) Ultra-high-speed photography was performed with the Imacon 200 camera. The camera takes 16 frames at rates up to 200 million frames per second. During the plate-impact tests on glass, the interframe time was between 200 and 300 ns with an exposure time of $20 \mathrm{~ns}$. The resolution of each of the images is $1200 \times 980$ pixels. The image intensifier of the camera uses $\mathrm{p} 46$ phosphor screen with a decay time of $200 \mathrm{~ns}$ to $10 \%$ of the fluorescence. This allows minimizing the ghosting effect when the sensors are being used in the second sweep. The first two tests used a Cooke camera with p43 phosphor screens. Due to ghosting it was virtually impossible to interpret properly the images observed. The Imacon camera was focused on the surface of the specimen (right surface as seen when looking from the gun side); that is, surface cracks should appear in-focus while interior defects should appear out-of-focus.

(3) Short pins were acquired from Dynasen (20 Arnold Place, Goleta, California 93117, USA.). The four shorting pins (Dynasen CA-1039-c) used in every test were arranged circumferentially around the target plate. One of the four shorting pin tips was located $0.7 \mathrm{~mm}$ above the surface of the target and the other three were flush with the target front surface. This configuration allowed measuring both the tilt of the projectile, see Table 1 , as well as the shock wave velocity. The shock velocity was measured using the PDV signal from the back of the target which had a common trigger with the pins. The internal gap distance to short the circuit in the pin was measured by Dynasen with a precision of $3 \mu \mathrm{m}$ and was taken into account when computing the tilt and shock wave velocity. The signal from the pins was recorded on a Tektronix oscilloscope at a rate of $1 \mathrm{GHz}$. At the typical velocities used in the tests presented in this work the error on the time of contact with a pin is estimated to be $\sim 10$ ns. This typically translates in an error in the tilt between 10 and $30 \%$, depending on the test considered.

\section{x-t Diagram}

Figure 3 shows the $\mathrm{x}-\mathrm{t}$ diagram calculated for the geometry selected. After impact a compression wave travels through the flyer plate and the target. This compression wave reaches the back of the flyer in $0.7 \mu$ s and the back of the target in $1.45 \mu \mathrm{s}$. The tensile waves should form the spall plane at $\mathrm{t} 2=2.15 \mu \mathrm{s}$, and the signal from the spall plane formation, i.e. the pullback signal from the back face of the target is expected to happen at $\mathrm{t} 3=2.86 \mu \mathrm{s}$. At the time of impact a release wave starts propagating spherically from the edge of the target. In the diagram it was assumed that the release wave traveled at the same speed than the shock wave: $5.6 \mathrm{~km} / \mathrm{s}$. The release wave takes longer $(\operatorname{tr}=3.47 \mu$ s) to reach the spall plane because it 
Table 1 Tests performed during the project that were recorded with the Imacon camera

\begin{tabular}{lllllll}
\hline Test \# & Impact velocity $(\mathrm{m} / \mathrm{s})$ & Interframe time $(\mathrm{ns})$ & Compressive stress $(\mathrm{GPa})$ & $\Delta \mathrm{V}(\mathrm{m} / \mathrm{s})$ & Tilt $(\mathrm{mrad})$ & Spall strength $(\mathrm{GPa})$ \\
\hline 4 & 212 & 200 & 1.30 & 2.8 & 2.4 & 0.017 \\
5 & 169 & 250 & 1.03 & 8.3 & 1.2 & 0.051 \\
6 & 130 & 250 & 0.80 & 25.2 & 2.1 & 0.154 \\
7 & 239 & 200 & 1.46 & 12.6 & 1.2 & 0.077 \\
8 & 283 & 200 & 1.73 & 7.8 & - & 0.048 \\
9 & 116 & 300 & 0.71 & 18.4 & N/A & 0.5 \\
10 & 351 & 200 & 2.15 & 1.8 & 0.113 \\
\hline
\end{tabular}

The exposure time was always $20 \mathrm{~ns}$

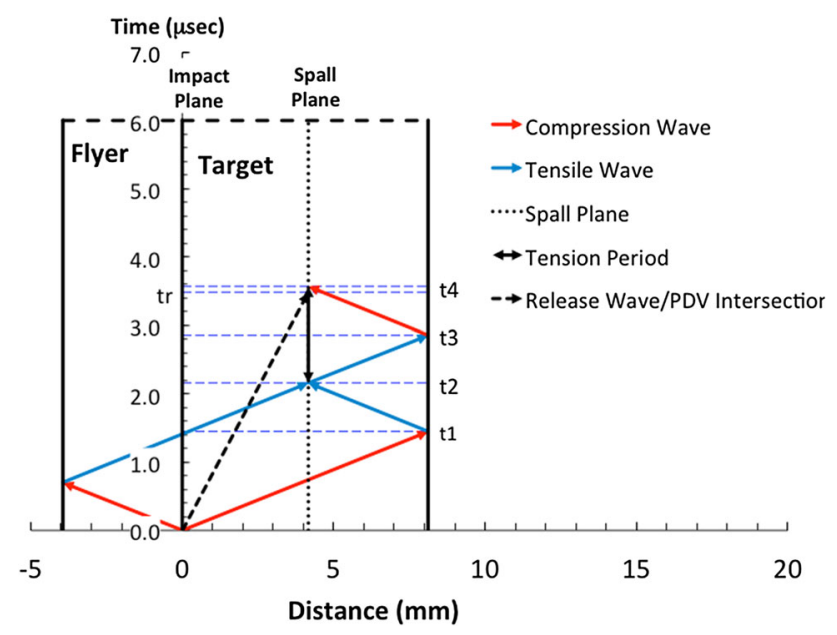

Fig. $3 \mathrm{x}-\mathrm{t}$ diagram for the geometry selected. $\mathrm{t} 1=1.45 \mu \mathrm{s}$, $\mathrm{t} 2=2.15 \mu \mathrm{s}, \mathrm{t} 3=2.86 \mu \mathrm{s}, \mathrm{t} 4=3.56 \mu \mathrm{s}, \mathrm{tr}=3.47 \mu \mathrm{s}$

travels a longer distance. Note that no failure wave was considered in this $\mathrm{x}-\mathrm{t}$ diagram. If the failure wave does not exist, the diagram shows that a spall plane could form before the release wave relaxes the target.

\section{Results}

Table 1 summarizes the experiments performed with Imacon images were recorded. The impact velocities ranged from 116 to $351 \mathrm{~m} / \mathrm{s}(0.7-2 \mathrm{GPa})$. All these impact velocities are well below the HEL of the glass which, depending on the reference, could be $7.5 \mathrm{GPa}$, see [2], or 8.7 GPa, see [6].

\section{Test with Elastic Release: Test\#9 $(116 \mathrm{~m} / \mathrm{s}$ or 0.71 GPa)}

The only test performed that provided a PDV signal consistent with elastic release was test 9. Figure 4(top) shows the pictures from the Imacon camera. The time (in microseconds) evolves from left to right and from upper to lower images. The time between images is $300 \mathrm{~ns}$. The shock appears in the second image at around $300 \mathrm{~ns}$ after trigger and moves to the right until reaching the back surface between 1.2 and $1.5 \mu \mathrm{s}$. At 1.8 and $2.1 \mu \mathrm{s}$ the reflection of the shock wave is observed traveling now from right to left. Image number 9 shows extensive failure that looks to be located on the surface (it seems in focus). This failure is probably due to surface effects.

The shock wave has a speed of approximately $6 \mathrm{~km} / \mathrm{s}$ as measured from the images. Test 9 had a pin very close to the surface of the target, only $0.16 \mathrm{~mm}$ above it (pin\#1). This allowed an accurate determination of the shock wave speed from the pin data and the time it takes the wave to reach the back of the target as provided by the PDV, see Fig. 4(bottom). Both the PDV and Imacon were triggered by pin\#1. The shock wave speed calculated in this way was $5.6 \mathrm{~km} / \mathrm{s}$.

Note that the pictures do not show any traces of damage until the last frame where a black opaque front appears on the left of the specimen. Computations were performed using EPIC [28] confirming that the timing and wave shapes are the ones expected. In particular the blue arrows show release effects coming from the boundaries.

As mentioned in the introduction, Cagnoux [2] also observed an elastic release on a similar test in Pyrex at the same compression stress level (0.7 GPa).

\section{Slowest Test without Elastic Release: Test\#6 (130 m/ s or $0.80 \mathrm{GPa})$}

Figure 5 top shows the high-speed photography pictures obtained for Test\#6. The first frame where the shock wave is visible is the frame with the time tag $0.75 \mu \mathrm{s}$. The shock wave is seen on the left of the specimen as a slightly inclined fine shadow. It moves to the right and crosses the whole specimen in approximately $1.75 \mu \mathrm{s}$. An important difference with Test\#9 is that at $\mathrm{t}=2.0 \mu \mathrm{s}$, what seems to be incipient damage appears on the left side of the specimen. The top-right image on Fig. 5 is a zoomed-in version 
Fig. 4 Test\#09 (top) images from Imacon camera, time in frames in microseconds. A tilt of $1.8 \mathrm{mrad}$ shows as a clearly inclined shock wave, (bottom) Signal recorded from the PDV. The arrows indicate target surface effects. Time in images is microseconds
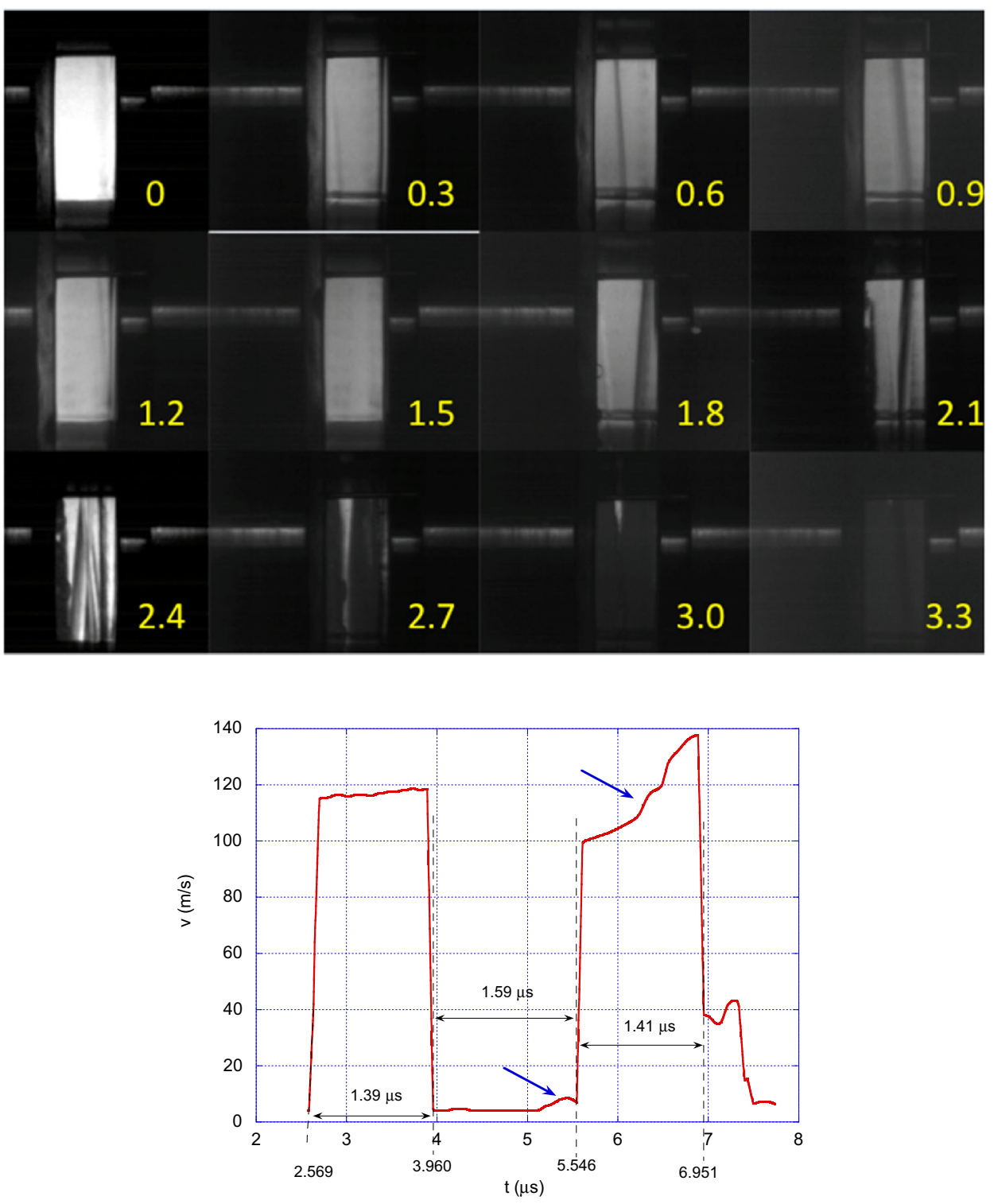

of the specimen for $\mathrm{t}=2.25 \mu$ s where a line of dark spots is developing on the left side of the specimen.

The reflection of the shock wave on the right side also happens at $\mathrm{t}=2.0 \mu$ s so that the wave starts traveling to the left. The wave seems to be stopping at $t=2.75 \mu \mathrm{s}$, probably because it is entering a damaged zone. This frame or the next one might be showing the spall plane, which, as expected, takes $1.45 \mu$ s to appear up in the PDV signal (Fig. 5, bottom).

If spall is computed using the classic equation:

$\sigma_{\text {spall }}=\frac{1}{2} \rho_{0} c \Delta v$

where $\rho_{0}$ is the density of glass, $c$ the sound speed, and $\Delta v$ the pullback velocity seen in the PDV signal; the spall strength in this case was $0.15 \mathrm{GPa}$. This value is certainly small for glass but, as observed by Cagnoux [2], the "apparent" spall strength decreases as the impact velocity decreases; i.e. the material that spalled was predamaged and its strength is very low.

\section{Test\#10: Highest Impact Velocity $(351 \mathrm{~m} / \mathrm{s}$ or 2.15 GPa)}

Figure 6 (top) shows the frames recorded during the test performed at the highest impact velocity $(351 \mathrm{~m} / \mathrm{s}, 2 \mathrm{GPa})$. The shock wave can be seen as a straight dark line in the frame at $\mathrm{t}=0.6 \mu \mathrm{s}$, on the left side of the specimen. On frame 5 the shock moves slightly to the right and it is already possible to see some damage developing behind the shock wave. This damage is apparent on frame six, at $\mathrm{t}=1.0 \mu \mathrm{s}$. Frame $8(\mathrm{t}=1.4 \mu \mathrm{s})$ shows what could be damage nucleation sites trailing the shock wave. These 
Fig. 5 Test\#6 (top) Image sequence of the shock wave propagating and failure, (topright) zoom of image of specimen at $2.25 \mu \mathrm{s}$, (bottom) PDV signal. Time in images is in microseconds
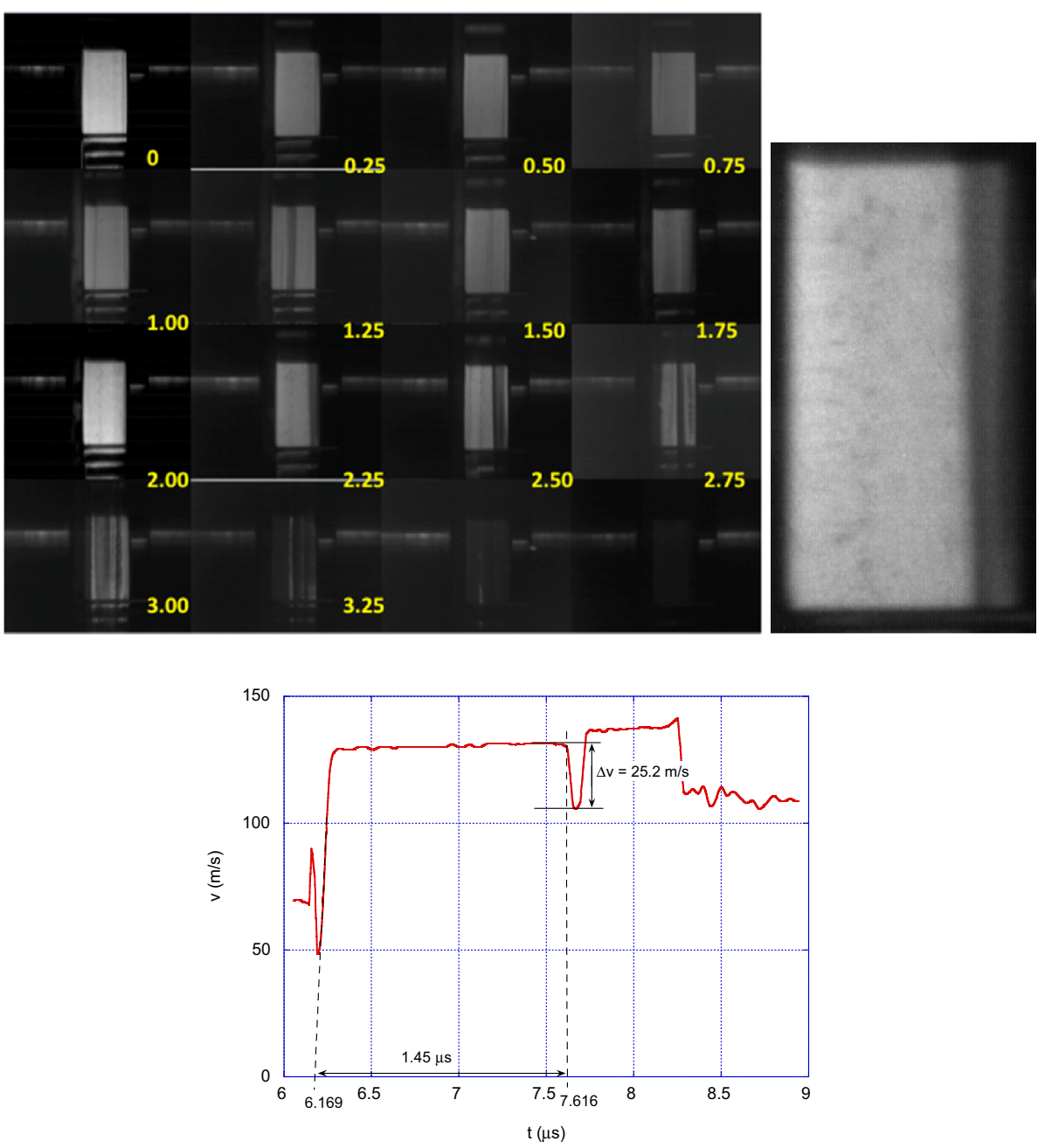

seem to coalesce and form a dark and opaque front that forms behind. The shock wave reaches the rear of the specimen (right side) between frames 10 and 11 (time $1.8-2.0 \mu \mathrm{s})$.

As shown in Fig. 6 (bottom) the spall signal is very weak, although it is happening at the time expected. Clearly, from looking at the high-speed pictures, it is possible to confirm that if any spall is happening it is happening inside damaged material.

\section{An Interesting Interpretation of the PDV Signal}

As mentioned in the methods section, the PDV works by analyzing the Doppler shift of the laser reflected from the back of the target. Since the glass specimens are transparent various methods were employed to make the back face reflective. In tests 4 through 8 , black ink was used and reflectivity was only partially achieved. The targets used in tests 9 and 10 were sputtered with gold which made the back face perfectly reflective.
A typical PDV recording for the specimens that used black ink is shown in Fig. 7. This spectrogram is very "rich" in information because it contains laser reflections from back of the target (BT), from the target/projectile interface (INT), from the back of the projectile (BP), and from the front of the projectile (FP). Before the impact occurs, only the signal from the front of the projectile is detected at the expected velocity $(212 \mathrm{~m} / \mathrm{s})$. Note the signal covers a band from approximately 180 to $240 \mathrm{~m} / \mathrm{s}$. The reason is that when analyzing the spectrogram there is a trade-off between accuracy on the velocity and accuracy on time. For the image shown, priority was given to increased sharpness in time. But the same signal was also analyzed in a way that it provided high accuracy on the velocity determination.

The impact occurs around $2.8 \mu$ s after the trigger. The velocity of the back of the projectile does not decrease until the shock wave travels through its thickness $(\sim 0.70 \mu \mathrm{s}$, see in Fig. 3 the $\mathrm{x}-\mathrm{t}$ diagram). As expected from the $\mathrm{x}-\mathrm{t}$ diagram, the velocity of the interface projectile/target is half the impact velocity and only lasts for $\mathrm{t} 1=1.45 \mu \mathrm{s}$ 
Fig. 6 Test\#10 (top) Image sequence of the shock wave propagating and failure, (bottom) PDV signal. Time in images is in microseconds
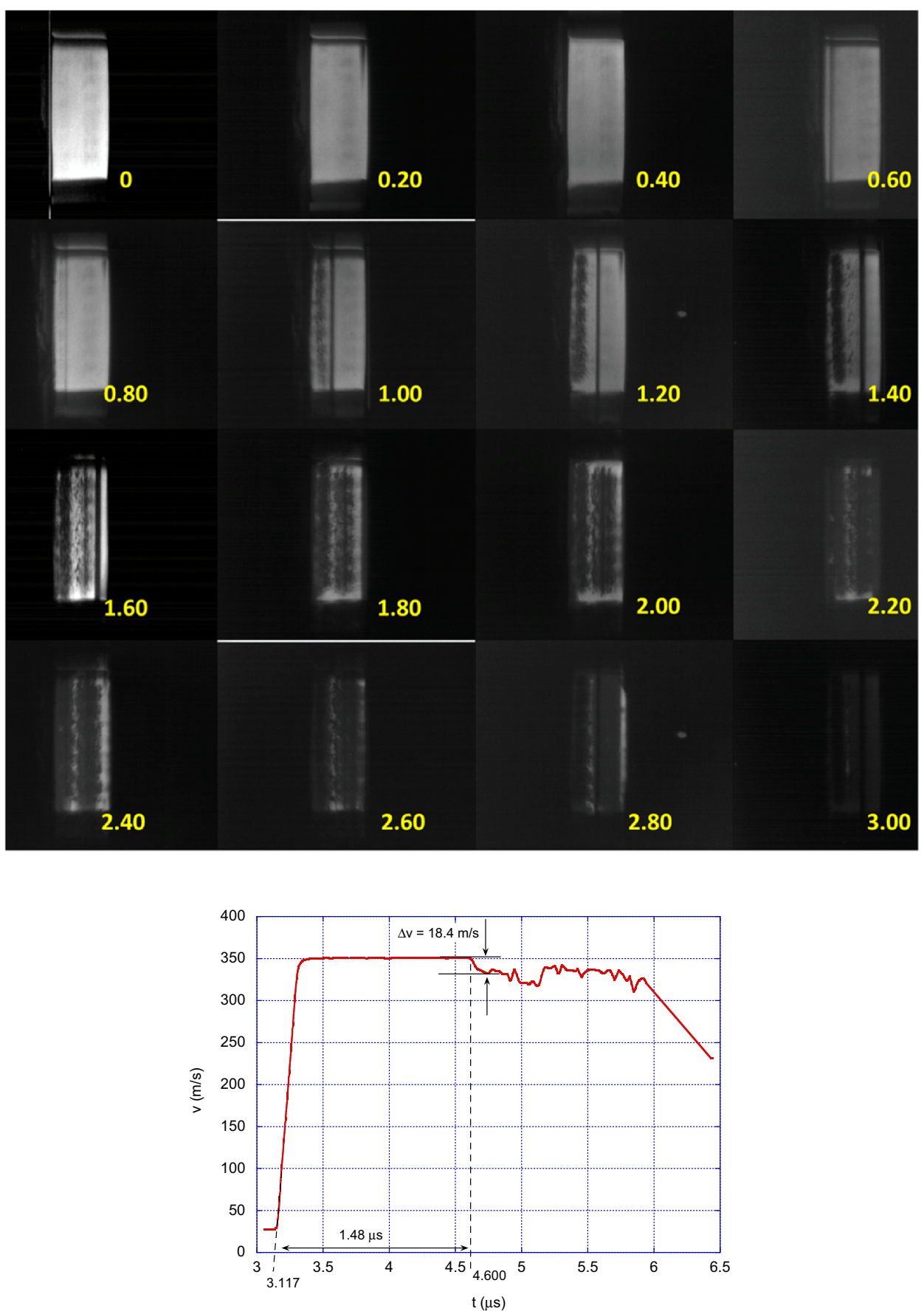

after impact. The back of the target does not start to move at the impact velocity until $\mathrm{t} 1$; i.e., at the time when the interface velocity drops to zero. The spall signal does seem to appear when expected, at $\mathrm{t} 3 \sim 2.9 \mu \mathrm{s}$. Note that Fig. 7 also shows a harmonic, probably coming from the interface signal. Harmonics are often seen in PDV signals and have a value that is twice, three times, etc. the velocity of the surface being tracked.

The laser reflecting from the projectile/target interface goes twice through the target before being detected by the
PDV. It is speculated in this work that, if the glass inside the target gets damaged with time, then the laser light might be dispersed and hence its intensity would decrease with time. This would support that the damage observed in the ultra-high-speed images is interior and not on the surface of the glass.

Figure 8 shows, for all the tests, the power being detected by the PDV, but only for the signal that is bouncing back from the projectile/target interface. The plots are presented in order of increasing impact velocity. 


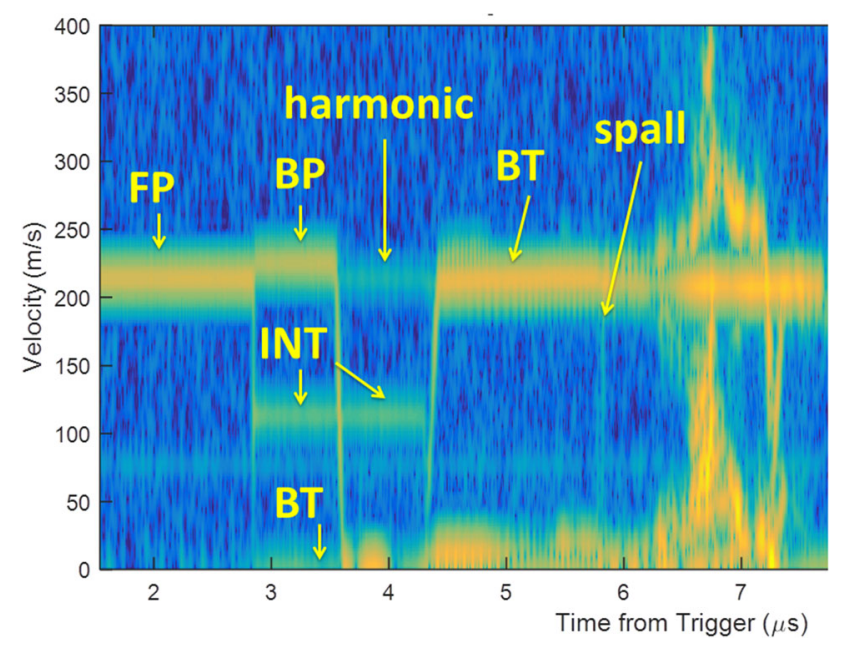

Fig. 7 Spectrogram for test 4 (impact velocity $212 \mathrm{~m} / \mathrm{s}$, stress $1.3 \mathrm{GPa}$ ) showing the signals from the different surfaces. $F P$ front of projectile, $B P$ back of projectile, $I N T$ interface projectile/target, $B T$ back of target

All the plots show a downward trend that indicates that the power received by the PDV decreases with time. This is consistent with having damage increasing with time in the target; i.e., the laser being increasingly dispersed as damage progresses. Although it is well known that the index of refraction depends on the compression of the glass, the authors of this paper cannot think of any mechanism other than failure for this effect. Note that at the highest velocities, the drop in the power is faster, implying that damage progresses faster in the specimen.

\section{EPIC Computations}

The hydrocode EPIC [28] was used to model the flyer-plate impact experiments to predict the spall behavior as well as to try to reproduce the damage observed in the experiments. Note that no calibration was performed based on the plate impact results presented above. Rather, the model was previously calibrated in recent work by Holmquist and Wereszczak [23], who inferred the spall strength in a borosilicate glass to be approximately $1.2 \mathrm{GPa}$ determined from laser impact experiments and computational analysis. It is important to note that the laser shock is only $20 \mathrm{~ns}$ long versus typically $1 \mu \mathrm{s}$ in plate impact and hence the calibration may be accessing different deformation mechanisms than the tests performed here.

Although Holmquist's and Wereszczak work [23] computed damage (spall) in a location similar to that observed in the experiment, there was concern because the computed morphology was not penny shaped, as exhibited in the laser-shock experiment. Further work was performed herein to address the difference in damage morphology.
Figure 9 presents damage in a 19-mm borosilicate target after a 5.1-GPa laser loading. The result on the far left is the damage produced using a spall stress of $1.20 \mathrm{GPa}$ and a time to fail $\left(\mathrm{t}_{\mathrm{fail}}\right)$ of $0.4 \mu \mathrm{s}$ taken from Holmquist and Wereszczak [23]. Time to fail is a feature in the Holmquist-Johnson (HJ) glass model [29] that fails the material over a specified time $\left(t_{\text {fail }}\right)$. When damage equals 1.0 it takes $t_{\text {fail }}$ to transition from the intact strength curve to the failed strength curve, which tends to inhibit the propagation of failure. The five computations shown on the right side of Fig. 9 present target damage as a function of spall strength and do not use the time to fail feature (here failure occurs instantaneously, $t_{\text {fail }}=0$ ). When the time to fail feature is not used, damage is penny shaped, in much better agreement with the experiment. Note that when the spall strength is between 1.20 and $1.35 \mathrm{GPa}$ the damage morphologies are similar and in reasonable agreement with the experiment, but when the strength is decreased to $1.11 \mathrm{GPa}$, damage also occurs near the front surface and when the strength is increased to $1.43 \mathrm{GPa}$ there is no damage anywhere in the target.

Figure 10 presents another laser impact test where the loading stress was $8.1 \mathrm{GPa}$ and the target was $19-\mathrm{mm}$ thick. A cross section of the top $7 \mathrm{~mm}$ of the post-mortem target is shown on the right side of Fig. 10 and shows significant damage (there was no damage in the lower $12 \mathrm{~mm}$ of the target and no rear surface spall). The left side of Fig. 10 shows two computed results, one using a spall strength of $1.20 \mathrm{GPa}$ and the other a spall strength of $1.27 \mathrm{GPa}$ (both use $t_{\text {fail }}=0$ ). The result using $1.20 \mathrm{GPa}$ produces rear surface spall (in disagreement with the experiment), the result using $1.27 \mathrm{GPa}$ does not. Figure 11 presents a comparison of the experiment and the computed result using similar scales (for a spall strength of $1.27 \mathrm{GPa}$ ) and are in agreement inasmuch as they both produce steep cone cracks, a failure plane and no rear surface spall. Computations using a spall strength of $1.27 \mathrm{GPa}$ and $\mathrm{t}_{\text {fail }}=0$ produce results that are in good agreement with both the $5.1 \mathrm{GPa}$ and the $8.1 \mathrm{GPa}$ laser spall experiments and will be used in all the following computations.

Figure 12 presents computed results showing damage in the plate-impact test for impact velocities of $\mathrm{V}=150,180$, 190, 200 and $210 \mathrm{~m} / \mathrm{s}$. The model does not predict damage in the target for $\mathrm{V}=150$ and $180 \mathrm{~m} / \mathrm{s}$ and spall did not occur. Although there is severe damage in the target when $\mathrm{V}=190$ and $200 \mathrm{~m} / \mathrm{s}$ classic spall did not occur.

The damage is a result of the release wave from the lateral edge of the target interacting with the release waves from the rear surfaces of the target and impact flyer, initiating damage midway through the target, approximately $12 \mathrm{~mm}$ from the axis of symmetry. This is demonstrated in Fig. 13 where damage is presented at $\mathrm{t}=2.4,2.6,2.8,3.0$, and $3.2 \mu$ s after impact for $\mathrm{V}=190 \mathrm{~m} / \mathrm{s}$. The tensile stress 
Fig. 8 Power measured from the projectile/target interface signal recorded by the PDV. The graphs are placed in order of smaller to larger impact velocities: a Test $06(130 \mathrm{~m} / \mathrm{s})$, b Test $05(169 \mathrm{~m} / \mathrm{s})$, c Test 04 $(212 \mathrm{~m} / \mathrm{s})$, d Test $03(215 \mathrm{~m} / \mathrm{s})$, e Test $07(239 \mathrm{~m} / \mathrm{s})$, f Test 08 $(283 \mathrm{~m} / \mathrm{s})$, see Table 1 for pressures attained in the different tests
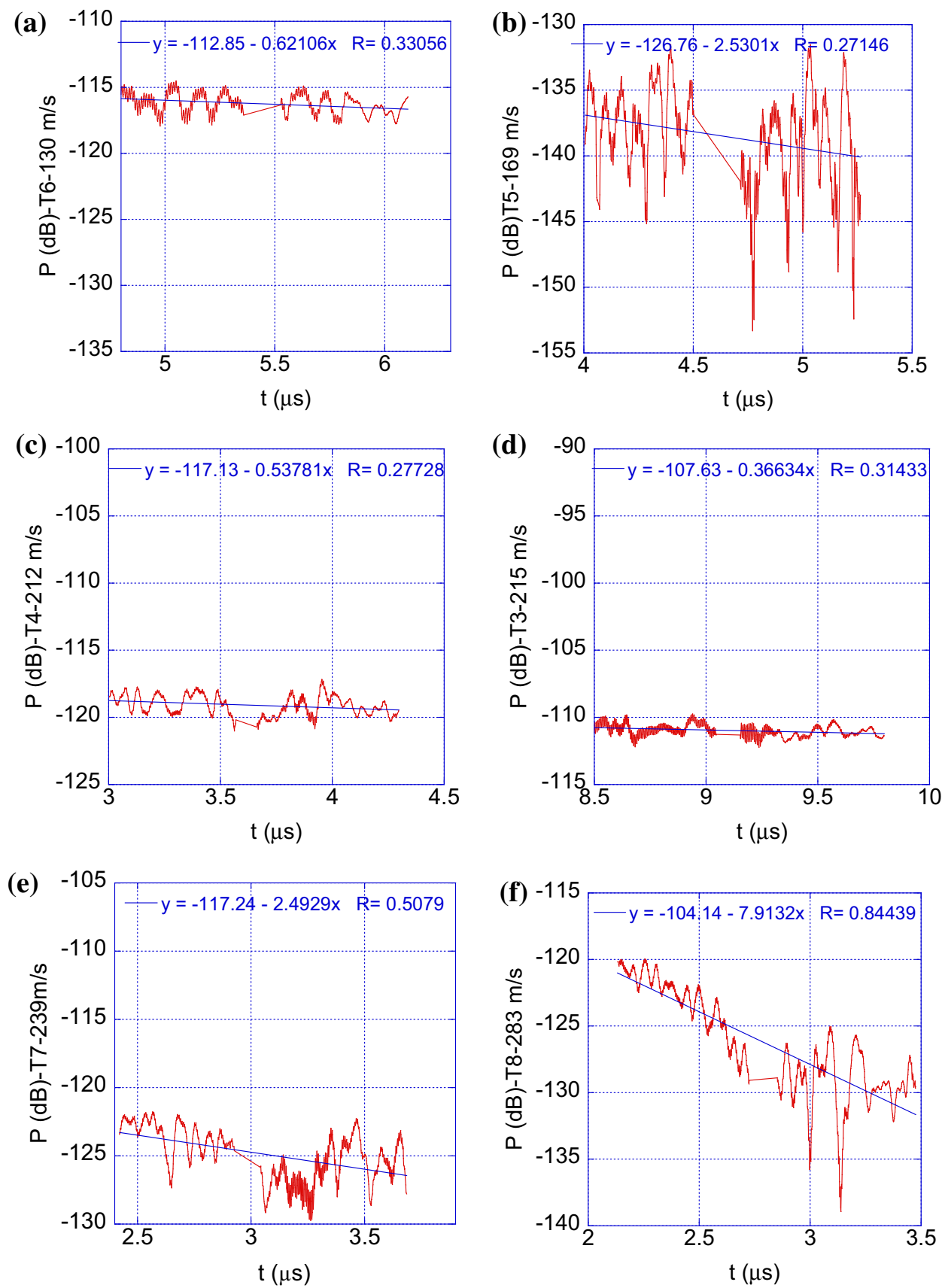

created from the release waves from the rear surfaces of the target and the flyer are not sufficient to create spall, but when combined with the release waves from the lateral edge results in a tensile stress sufficient to cause damage. When $\mathrm{V}=210 \mathrm{~m} / \mathrm{s}$, classic spall occurs. These results were confirmed by 1D uniaxial strain computations that produced spall at $\mathrm{V}=210 \mathrm{~m} / \mathrm{s}$ and no spall (complete elastic release) at $\mathrm{V}=200 \mathrm{~m} / \mathrm{s}$.

EPIC seems to be underpredicting the damage as observed with the high-speed photography. This is not surprising because there is probably time dependence in the failure of borosilicate glass; i.e., damage in glass not only depends on the magnitude of the load but also on how long the load is being applied. A typical laser shock experiment provides a pulse load length of $\sim 20 \mathrm{~ns}$, while for the plate impact test presented above the loading time is $\sim 1 \mu \mathrm{s}$, which is two orders of magnitude larger. The difference in the results may well be justified in this context. This matter deserves further investigation. Because of the interaction between the failure front and the release waves from edges and the back of the target, accurately predicting damage and damage evolution without a more sophisticated 


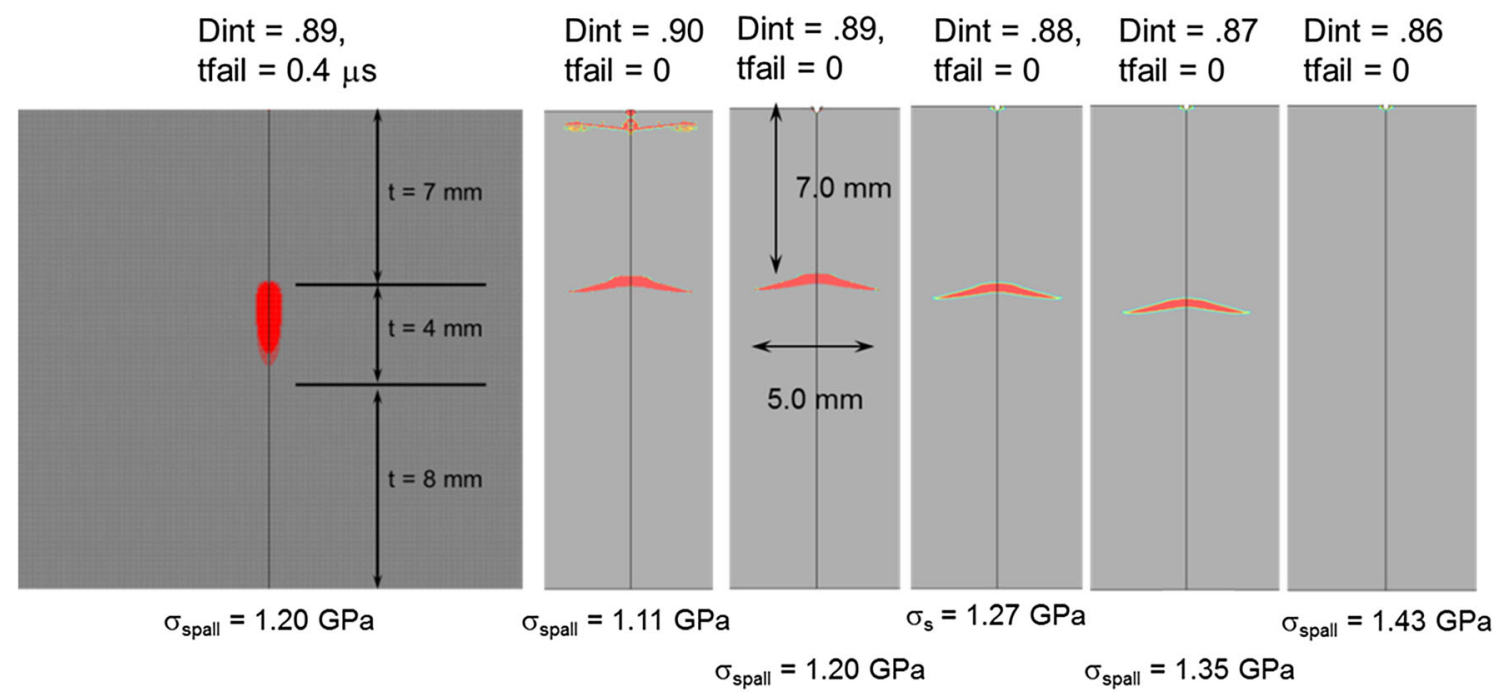

Fig. 9 Damage produced from a 5.1-GPa laser loading experiment. Damage is shown as a function of spall strength and time to fail ( $\left.t_{\text {fail }}\right)$
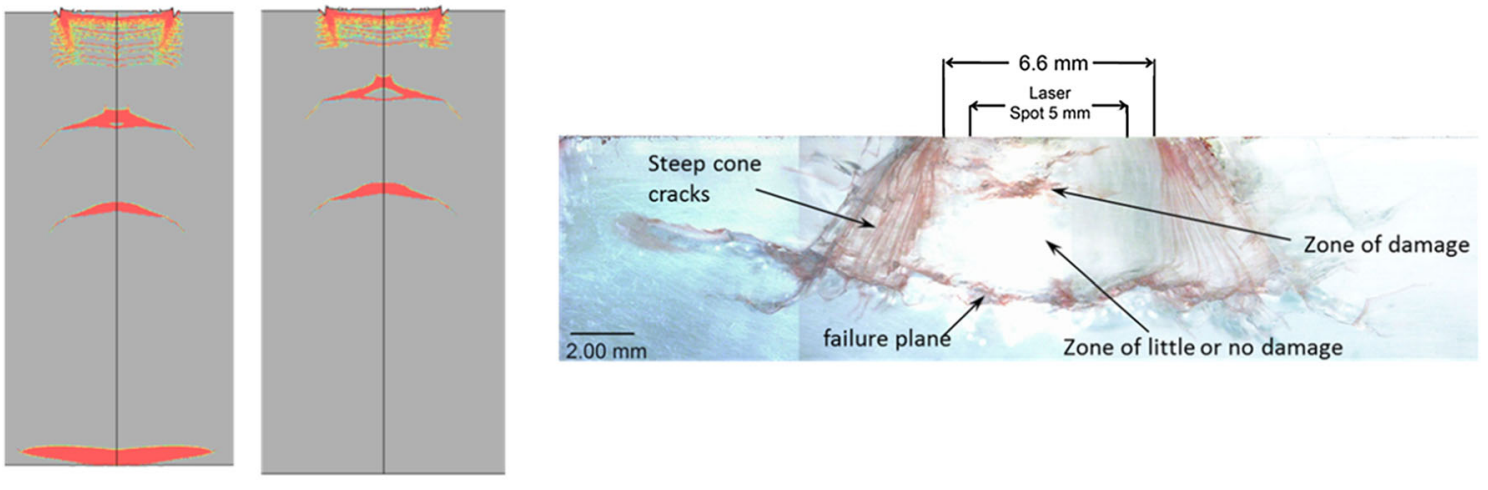

$$
\sigma_{\text {spall }}=1.20 \mathrm{GPa} \quad \sigma_{\text {spall }}=1.27 \mathrm{GPa}
$$

Fig. 10 Computed and experimental results for the 8.1-GPa laser loading test. The left shows computed results, showing damage, for a spall strength of $1.20 \mathrm{GPa}$ and for $1.27 \mathrm{GPa}$. The right shows the top $7 \mathrm{~mm}$ of the damaged target

Fig. 11 A comparison of damage produced in the experiment and the computed result for a 8.1-GPa laser laoding. The computed result used a spall strength of 1.27 GPa and no time to fail $\left(\mathrm{t}_{\text {fail }}=0\right)$

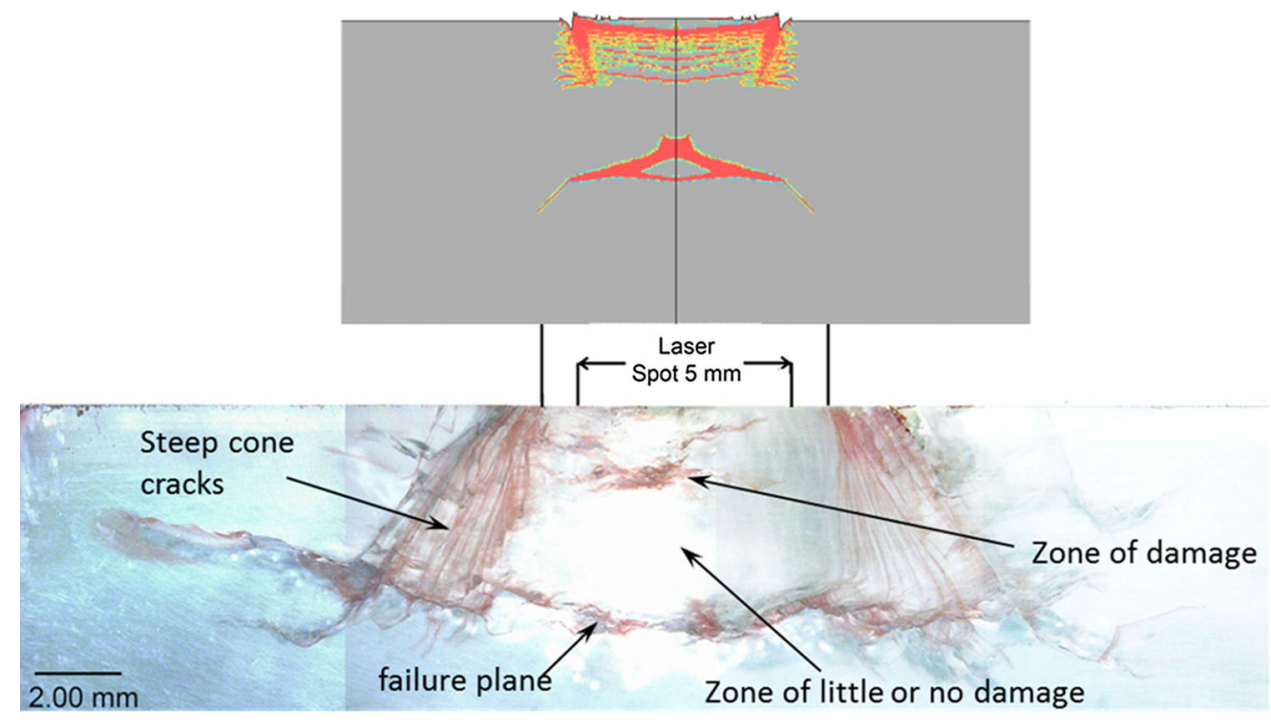


Fig. 12 Computed results showing damage for impact velocities of $\mathrm{V}=150,180,190$ 200 and $210 \mathrm{~m} / \mathrm{s}$. Classic spall only occurred for $\mathrm{V}=210 \mathrm{~m} / \mathrm{s}$
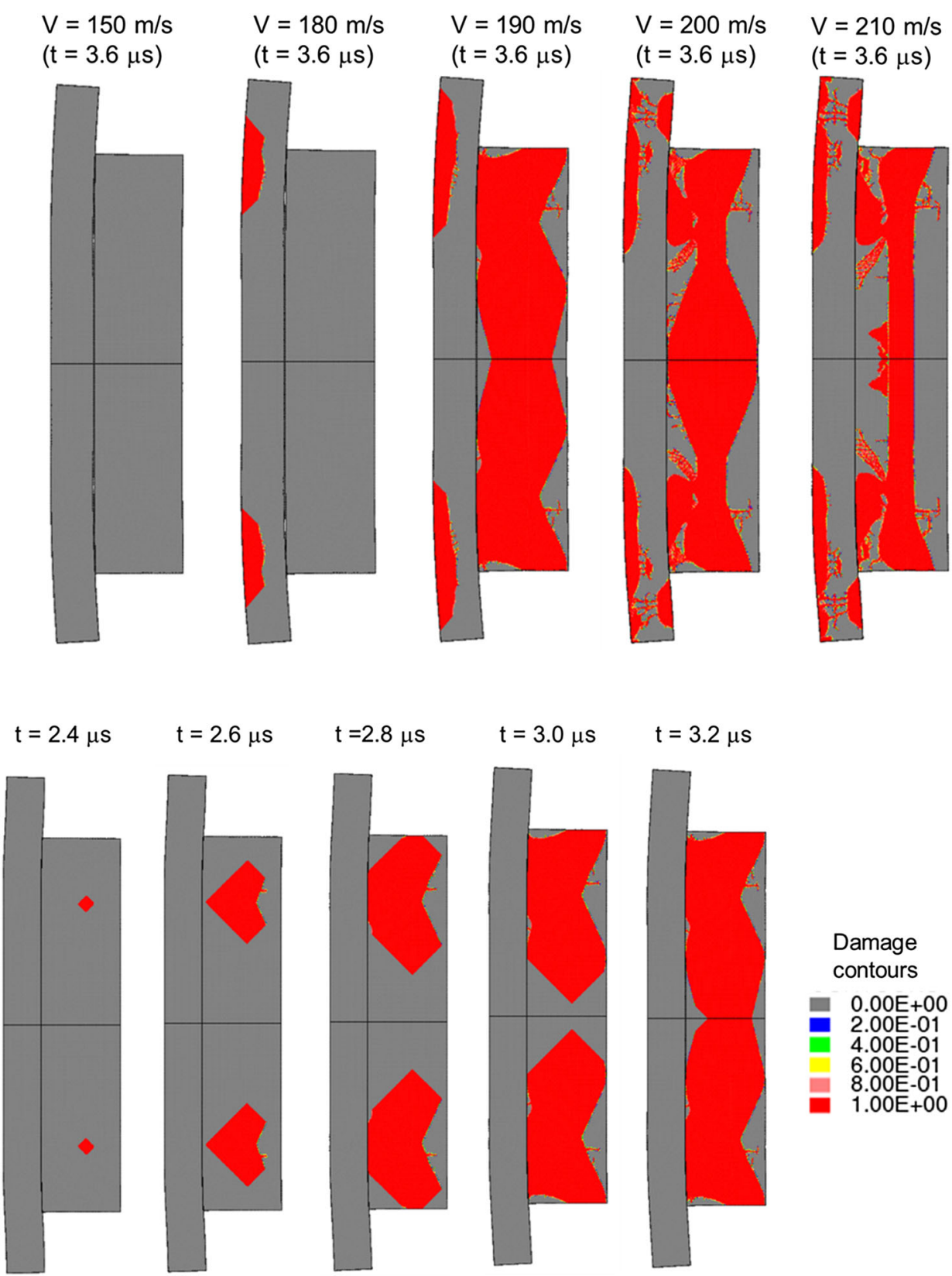
contours

Fig. 13 Damage shown at $\mathrm{t}=2.4,2.6,2.8,3.0$, and $3.2 \mu \mathrm{s}$ after impact. The impact velocity was $\mathrm{V}=190 \mathrm{~m} / \mathrm{s}$ (Color figure online)

damage model becomes virtually impossible. Nevertheless the value of $1.27 \mathrm{GPa}$ as the spall strength for glass can be probably be used as an upper limit for extremely short during pulses, i.e. for long stress pulses it is expected to be lower.

\section{Discussion and Conclusions}

Flyer-plate impact experiments have been conducted on a borosilicate glass using very-high-speed camera for visual observations, combined with PDV to measure velocities. The fact that the shock wave can easily be seen in the camera images validates in part the observational method. It is well known that the index of refraction of the glass changes with the pressure and that refraction gradients obscure the background light that goes through the glass. Additionally the shock wave velocity was measured using the pictures and the PDV providing similar results. The reflection of the shock wave can clearly be seen in every test but the highest velocity experiment (\#10), due to the extensive damage trailing the wave. In all the cases, a little after reflection from the rear surface, the shock wave "disappears", probably because of release waves from the boundaries and because the wave is traveling through damaged material and dissipating. 
The first important result of this work is the fact that we are seeing damage nucleating behind the shock wave at velocities possibly as slow as $130 \mathrm{~m} / \mathrm{s}$ (compressive stress $0.8 \mathrm{GPa}$ ) and for sure at an impact velocity of $170 \mathrm{~m} / \mathrm{s}$ (1 GPa stress). EPIC computations show damage starting at $190 \mathrm{~m} / \mathrm{s}$, which was calibrated using a laser spall experiments. These computations provide a spall strength of 1.27 GPa as an upper limit for pulses of $\sim 20 \mathrm{~ns}$ length. Longer pulses would probably spall the glass at smaller stresses.

The stresses generated by the low velocities used in this investigation are well below the HEL of the glass so the HEL cannot be interpreted as a threshold for damage. Clearly the damage grows faster at higher impact velocities; although, when the image is clean, it is not possible to say that the specimen is undamaged. It may be that the size of the damage is microscopic and undetectable with the method being used.

The speckled pattern (nucleation sites) behind the shock is very obvious at impact velocities of $169 \mathrm{~m} / \mathrm{s}$ and above. Since the camera was focused on the target surface, the fact that the speckles seem out of focus and happening at early time indicates that it is a phenomenon that may be happening inside the target and not on the surface. A description on how failure propagates through glass can be found in the book by Bourne [30], where it appears to be similar to what is seen in this work but, instead of a sharp black front, speckles trailing the shock wave are observed. This may be due to the small pressure behind the shock in our tests. Our fastest shot clearly shows that the trailing failure gets darker and sharper. This seems to be confirmed by a new diagnostic technique where the power of the PDV signal reflected from the interface projectile/target was recorded. The fact that the power decreases with time supports that the laser light is being dispersed, hence implying that damage is growing/coalescing inside the target.

Another interesting observation from the tests performed is that no recompression wave is detected with the PDV. The recompression wave is expected when the release wave from the back surface meets a failure front with lower impedance. This should create another reflection (compressive wave) that travels again to the right and meets the back of the target creating a small "bump" on the PDV signal (this interpretation of the "bump" is from Kanel). None of the tests performed during this program showed the recompression signal clearly. This is in agreement with what was observed by other researchers at low velocities. The reason might be that at these low impact velocities the recompression wave is too small to be seen and is lost in the noise. It is speculated that the absence of the recompression signal may have confused researchers by thinking that the failure was not occurring at these low impact velocities.
EPIC computations were performed and compared to the results from the experiments. EPIC seems to be underpredicting the damage threshold on the glass. These results are under investigation.

Acknowledgments The author Sidney Chocron wishes to thank fruitful discussions with Dr. Stephan Bless who provided great insight in the interpretation of the PDV signals and shock wave propagation in glass. Sidney also thanks email correspondence and conversations with Dr. Zvi Rosenberg. Zvi provided great insight on HEL, spall, and failure wave studies on glass that he is so familiar with. The authors would like to acknowledge funding from Southwest Research Institute, under the Internal Research and Development Program.

\section{References}

1. Walley SM (2013) An introduction to the properties of silica glass in ballistic applications. Strain 50:470-500

2. Cagnoux J (1982) Rupture Par Ecaillage d'un verre de borosilicate. J Phys Colloq 43(C9):C9-591-C9-594

3. Cagnoux J, Longy F (1988) Spallation and shock-wave behaviour of some ceramics. J Phys Colloq 49(C3):C3-3-C3-10

4. Kanel GI, Molodets AM, Dremin AN (1978) Investigation of singularities of glass strain under intense compression waves. Transl. Fiz. Goreniya i Vzryya 13(6):772-777

5. Cagnoux J (1982) Shock-wave compression of a borosilicate glass up to 170 kbar. AIP Conf Proc 78:392-396

6. Alexander CS, Chhabildas LC, Reinhart WD, Templeton DW (2008) Changes to the shock response of fused quartz due to glass modification. Int J Impact Eng 35(12):1376-1385

7. Yeshurun Y, Rosenberg G, Rosenberg Z (1985) Measurements of compressive and tensile wave in a shock loaded pyrex glass. Shock waves in condensed matter. AIP, New York, pp 431-434

8. Kanel GI, Rasorenov SV, Fortov VE (1992) The failure waves and spallations in homogeneous brittle materials. In: Schmidt SC, Dick RD, Forbes JW, Tasker DG (eds) Shock compression of condensed matter 1991. North-Holland, Amsterdam

9. Brar NS, Bless SJ, Rosenberg Z (1991) Impact-induced failure waves in glass bars and plates. J Appl Phys 59(26):3396-3398

10. Bourne NK, Forde LC, Millett JCF, Field JE (1997) Impact and penetration of a borosilicate glass. J Phys IV 7:1-6

11. Brar N, Rosenberg Z, Bless S (1991) Spall strength and failure waves in glass. J Phys IV 1:3-8

12. Bourne NK, Rosenberg Z, Field JE (1995) High-speed photography of compressive failure waves in glasses. J Appl Phys 78(6):3736-3739

13. Bourne NK, Rosenberg Z, Mebar Y, Obara T, Field JE (1994) A high-speed photographic study of fracture wave propagation in glasses. J Phys 4:C8-635-C8-640

14. Espinosa H, Xu Y, Brar NS (1997) Micromechanics of failure waves in glass: I, experiments. J Am Ceram Soc 80(8):2061-2073

15. Brar NS, Bless SJ (1992) Failure waves in glass under dynamic compression. High Press Res 10:773-784

16. Rosenberg Z, Yaziv D, Bless S (1985) Spall strength of the shock-loaded glass. J Appl Phys 58(8):3249-3251

17. Ginzburg A, Rosenberg Z (1998) Using reverberation techniques to study the properties of shock loaded soda-lime glass. Shock compression of condensed matter 1997, vol 529. American Institute of Physics, New York, pp 1-4

18. Chocron S, Dannemann KA, Walker JD, Nicholls AE, Charles J, Anderson E (2007) Constitutive model for damaged borosilicate glass under confinement. J Am Ceram Soc 90(8):2549-2555 
19. Tolman J, Bless S, Levinson S (2009) Dynamic strength measurements on transparent materials using bar impact. In: Proceedings of the SEM annual conference, vol 2

20. Partom Y (1998) Modeling failure waves in glass. Int J Impact Eng 21(9):791-799

21. Bourne NK, Rosenberg Z (1996) The dynamic response of sodalime glass. AIP Conf Proc 370(1996):567-572

22. Anderson CE, Orphal DL, Templeton DW (2004) Re-examination of the requirements to detect the failure wave velocity in $\mathrm{SiC}$ using penetration experiments. In: Proceedings of shock compression of condensed matter-2003, pp 707-770

23. Holmquist TJ, Wereszczak AA (2014) The internal tensile strength of a borosilicate glass determined from laser shock experiments and computational analysis. Int J Appl Glass Sci 5:345-352

24. Kanel GI (2006) Failure waves in shock-compressed glasses. In: Shock compression of condensed matter 2005, pp 870-875
25. Brar NS (2000) Failure waves in glass and ceramics under shock compression. In: Shock compression of condensed matter 1999, pp 601-606

26. Wereszczak AA, Anderson CE (2014) Borofloat and Starphire float glasses: a comparison. Int J Appl Glass Sci 5(4):334-344

27. Strand OT, Goosman DR, Martinez C, Whitworth TL, Kuhlow WW (2006) Compact system for high-speed velocimetry using heterodyne techniques. Rev Sci Instrum 77:083108

28. Johnson GR, Stryk RA, Holmquist TJ, Beissel SR (1997) Numerical algorithms in a Lagrangian hydrocode. Wright Laboratory, Eglin Air Force Base Technical Report WL-TR-1997-7039

29. Holmquist TJ, Johnson GR (2011) A computational constitutive model for glass subjected to large strains, high strain rates and high pressures. J Appl Mech 78:051003

30. Bourne NK (2013) Materials in mechanical extremes, fundamentals and applications. Cambridge University Press, Cambridge, UK 